\title{
Decreased phosphatidylcholine plasmalogens - A putative novel lipid signature in patients with stable coronary artery disease and acute myocardial infarction
}

\author{
Iryna Sutter $^{\text {a, b, } 1 \text {, Roland Klingenberg }}{ }^{\mathrm{c}, 1}$, Alaa Othman ${ }^{\text {a, e }}$, Lucia Rohrer ${ }^{\mathrm{a}}$, \\ Ulf Landmesser ${ }^{\text {b, c, d, Dierik Heg }}{ }^{\mathrm{e}}$, Nicolas Rodondi ${ }^{\mathrm{f}}$, Francois Mach ${ }^{\mathrm{g}}$, \\ Stephan Windecker ${ }^{\mathrm{h}}$, Christian M. Matter ${ }^{\mathrm{b}, \mathrm{c}}$, Thomas F. Lüscher ${ }^{\mathrm{b}, \mathrm{c}}$, \\ Arnold von Eckardstein ${ }^{\mathrm{a}, \mathrm{b}, \mathrm{i}}$, Thorsten Hornemann ${ }^{\mathrm{a}, \mathrm{b}, \mathrm{i}, \text { * }}$ \\ ${ }^{a}$ Institute of Clinical Chemistry, University and University Hospital of Zurich, Zurich, Switzerland \\ ${ }^{\mathrm{b}}$ Competence Center for Integrated Human Physiology, University of Zurich, Zurich, Switzerland \\ ${ }^{\mathrm{c}}$ Department of Cardiology and Cardiovascular Research, University Heart Center, University Hospital Zurich, Zurich, University of Zurich, Switzerland \\ d Department of Cardiology, Charité Universitätsmedizin Berlin, Berlin, Germany \\ e Institute of Social and Preventive Medicine, Clinical Trials Unit, University of Bern, Bern, Switzerland \\ ${ }^{\mathrm{f}}$ Department of Internal Medicine, University Hospital Bern, Bern, Switzerland \\ ${ }^{g}$ Cardiology, University Hospital Geneva, Switzerland \\ h Cardiovascular Center, University Hospital Bern, Switzerland \\ ${ }^{\mathrm{i}}$ Competence Center for Systems Physiology and Metabolic Diseases, ETH Zurich and University of Zurich, Zurich, Switzerland
}

Keywords:

Glycerophospholipids

Sphingolipids

Coronary artery disease

Acute myocardial infarction

\begin{abstract}
A B S T R A C T
Objective: Glycerophospholipids and sphingolipids are structurally heterogeneous due to differences in the $\mathrm{O}$ - and $\mathrm{N}$-linked fatty acids and head groups. Sphingolipids also show a heterogeneity in their sphingoid base composition which up to now has been little appreciated. The aim of this study was to investigate the association of certain glycerophospholipid and sphingolipid species with stable coronary artery disease (CAD) and acute myocardial infarction (AMI).

Methods: The lipid profile in plasma from patients with stable CAD $(n=18)$ or AMI $(n=17)$ was compared to healthy subjects $(\mathrm{n}=14)$. Sixty five glycerophospholipid and sphingolipid species were quantified by LC-MS. The relative distribution of these lipids into lipoprotein fractions was analyzed. Results: In the CAD cohort, 45 glycerophospholipid and sphingolipid species were significantly lower compared to healthy controls. In the AMI group, 42 glycerophospholipid and sphingolipid species were reduced. Four PC plasmalogens (PC33:1, PC33:2, PC33:3 and PC35:3) showed the most significant difference. Out of eleven analyzed sphingoid bases, four were lower in the CAD and six in the AMI group. Sphingosine-1-phosphate (S1P) levels were reduced in the AMI group whereas an atypical C16:1 S1P was lower in both groups. Phosphatidylcholine and sphingomyelin species were exclusively present in lipoprotein particles, whereas lysophosphatidylcholines were mainly found in the lipoprotein-free fraction. The observed differences were not explained by the use of statins as confirmed in a second, independent cohort.

Conclusions: Reduced levels of four PC plasmalogens (PC33:1, PC33:2, PC33:3 and PC35:3) were identified as a putatively novel lipid signature for CAD and AMI.
\end{abstract}

* Corresponding author. Inst. for Clinical Chemistry, University Hospital Zurich, Raemistrasse 100, CH-8091, Zurich, Switzerland.

E-mail address: thorsten.hornemann@usz.ch (T. Hornemann).

1 Both authors contributed equally.

\section{Introduction}

Coronary artery disease (CAD) is a major cause of death and morbidity in the western world [1]. CAD is caused by the gradual development of atherosclerotic plaques in the arterial wall. A rupture or erosion of plaques with subsequent total occlusion of an 
epicardial coronary artery may lead to acute myocardial infarction (AMI) [2,3]. Recent data suggest that beyond established lipids risk factors like cholesterol and triacylglycerols also glycerophospholipids and sphingolipids contribute to atherogenesis [4,5]. Plasma glycerophospholipids and sphingolipids are mainly associated with lipoprotein particles [6-8] and form a lipid monolayer around the hydrophobic core of triacylglycerols and cholesteryl esters. Prior work identified distinct lipid profiles of plasma, serum or lipoprotein particles in patients with CAD [9-13]. Hundreds of different glycerophospholipid and sphingolipid species were identified in plasma lipoproteins and atherosclerotic plaques $[14,15]$. In particular sphingolipids are structurally diverse because of a multiplicity in O-linked head groups and N-linked fatty acids. Sphingolipids also show a significant heterogeneity in the sphingoid base composition which yet has been only little appreciated. Sphingolipid de-novo formation is initiated by the conjugation of $\mathrm{L}^{-}$ serine and acyl-CoA, a reaction catalyzed by the enzyme serine palmitoyltransferase (SPT). Sphingoid bases, the product of this reaction are the common structural feature for all sphingolipid species. The most abundant sphingoid bases in plasma is $C_{18}$ sphingosine, but SPT can also form other sphingoid bases in the range of $C_{14}-C_{20}$ [16]. Under certain conditions, SPT also metabolizes L-alanine and glycine which forms an atypical category of 1deoxysphingolipids (1-deoxySLs) [17,18] which were shown to be elevated in several metabolic conditions.

The aim of this study was to identify novel plasma lipid signatures for patients with CAD or AMI.

\section{Materials and methods}

\subsection{Patients}

Healthy subjects $(n=14$ ) were recruited from the local blood bank. Exclusion criteria were the use of cardiovascular medication, positive cardiovascular family history, smoking (including cessation of smoking within 2 years prior to study enrollment), history of hypertension, elevated total cholesterol $(\geq 5.0 \mathrm{mmol} / \mathrm{L})$, BMI $>30 \mathrm{~kg} / \mathrm{m} 2$, history of diabetes mellitus, evidence of relevant vascular or structural heart disease and/or a reduced LVEF $(<55 \%)$ on echocardiogramm. The stable CAD group $(n=18)$ was selected from patients with angiographically documented coronary artery stenosis $\geq 50 \%$. Exclusion criteria comprised an ACS within the preceding 6 months, systemic infectious, inflammatory or autoimmune disease, known severe renal dysfunction (serum creatinine $>220 \mu \mathrm{mol} / \mathrm{L}$ ), known severe hepatic dysfunction (3x ULN for LFTs), neoplasm or other life-threatening disease with a life expectancy less than one year, extended surgery in the preceding 3 months and/or evidence of vascular or structural heart disease and/ or a reduced systolic LV function on echocardiogram or left ventricular angiogram.

Patients with AMI $(n=17)$ were selected from the Inflammation in Acute Coronary Syndromes cohort (SPUM-ACS, NCT01000701). SPUM-ACS inclusion criteria comprised both genders (aged $\geq 18$ years) presenting within five days (preferably within $72 \mathrm{~h}$ ) after onset of chest pain and a main diagnosis of STEMI, NSTEMI or unstable angina as described previously [19].

All patients gave written informed consent and the study was conducted with approval from the local ethics committee (EK1680).

\subsection{Sampling of plasma}

Blood was drawn from the antecubital vein in healthy subjects and from the femoral/radial artery at the time of diagnostic coronary angiography in patients with CAD or AMI, respectively.
Samples were centrifuged at $2700 \mathrm{~g}$ for $10 \mathrm{~min}$ at room temperature to obtain plasma, frozen and stored in aliquots at $-80^{\circ} \mathrm{C}$ until serial measurement (no prior freeze-thaw cycles). To avoid interassay differences all samples were extracted and analyzed in a single batch. The person who performed the analysis was blinded to the patient's data by the use of numbered ID codes.

\subsection{Clinical chemistry}

Plasma concentrations or activities of total cholesterol, triglycerides, LDL-C and HDL-C, glucose, creatine kinase, CK-MB, and troponin levels were determined by photometric tests or immunoassay by using the Cobas 8000 autoanalyzer from Roche diagnostics (Rotkreuz, Switzerland).

\subsection{Isolation of lipoproteins}

Human lipoprotein fractions and the lipoprotein-free fraction (LFF) were isolated from plasma of three healthy blood donors by stepwise ultracentrifugation, as described previously [20]. Purity of lipoprotein fractions was confirmed by SDS-PAGE and Coomassie Blue staining.

\subsection{Analysis of plasma glycerophospholipids and sphingolipids}

Six lipid classes including phosphatidylglycerols (PGs), phosphatidic acids (PAs), phosphatidylethanolamines (PEs), phosphatidylcholines (PCs), sphingomyelins (SMs) and ceramides/ hexosylceramides (Cers/HexCers) were analyzed. Lipids were quantified in relation to internal standards (200 ng) including PG(17:0/17:0), LPG(17:1/0:0), PA(14:0/14:0), LPA(17:0/0:0), PE(14:0/ 14:0), LPE(17:1/0:0), PC(14:0/14:0), PC(24:0/24:0), LPC(17:0/0:0), SM (d18:1/12:0) and Cer(d18:1/17:0) (Avanti Polar Lipids, Alabaster, AL, USA). $20 \mu \mathrm{l}$ plasma was extracted with $375 \mu \mathrm{l}$ of methanol/ chloroform $(2: 1 \mathrm{v} / \mathrm{v})$. After vortexing, $100 \mu \mathrm{l}$ water and $125 \mu \mathrm{l}$ chloroform were added, agitated (15 min), centrifuged $(16,100 \times g$ for $5 \mathrm{~min}$ at $25{ }^{\circ} \mathrm{C}$ ) and the lower phase collected. Again $250 \mu \mathrm{l}$ chloroform was added, agitated (15 $\mathrm{min}$ ) and centrifuged. The lower phases were combined and evaporated under a stream of nitrogen. Analysis was done on a TSQ Quantum Access triple quadrupole connected to a Rheos 2200 pump. Separation was done on a diol silica-based column (QS Uptisphere $6 \mathrm{OH}, 150 \times 2.1 \mathrm{~mm}$, $5 \mu \mathrm{m}$ ). Mobile phase (A) was hexane/isopropanol/water (70:30:2 v/ v) including $15 \mathrm{mM} \mathrm{NH}_{4} \mathrm{COOH}$; and (B) isopropanol/water (50:2 v/ v) including $15 \mathrm{mM} \mathrm{NH} \mathrm{m}_{4} \mathrm{COOH}$ at a flow rate of $0.35 \mathrm{ml} / \mathrm{min}$. Gradient was 0-7 min A/B (\%) 80/20; 8-10 min A/B (\%) 60/40, 11-23 $\mathrm{min} \mathrm{A/B} \mathrm{( \% )} \mathrm{40/60} \mathrm{and} \mathrm{25-30} \mathrm{min} \mathrm{A/B} \mathrm{( \% )} \mathrm{80/20.} \mathrm{Dried}$ material was reconstituted in $200 \mu \mathrm{l}$ of mobile phases $A(80 \%)$ and $B$ (20\%) an injected $(10 \mu \mathrm{l})$ into the LC-MS. Neutral loss and precursor scans were used to detect specific glycerophospholipids and sphingolipids. A neutral loss scan of $m / z 115$ and 189 from $\left[\mathrm{M}+\mathrm{NH}_{4}\right]^{+}$ions was used for the analysis of PA and PG lipids. Precursor ion scan of $m / z 184$, specific for phosphocholine was used for PC, SM and LPC lipids. Neutral loss scan of $m / z 141$ was used for PEs and precursor scanning of $m / z 264$ was applied for Cer and HexCer species. Data were analyzed in Xcalibur (version 2.0.6, Thermo Scientific). Identification of molecular species was performed by lipid mass spectrum analysis software (LIMSA) [21].

The assignment of glycerophospholipids includes total numbers of carbons and double bonds in two acyl chains. The SM assignment comprises total numbers of carbons and double bonds in the sphingoid base and $\mathrm{N}$-linked fatty acid. Hexosylceramide species include isomeric glucosyl- and galactosylceramides. For identified Cers and HexCers, molecular structures are reported. Quantification was done in relation to the respective internal standards. 


\subsection{Quantification of sphingosine-1-phosphate species}

Sphingosine-1-phosphates (S1Ps) in plasma samples were determined by LC-MS as described previously [22]. Concentrations of S1P species were measured in $25 \mu \mathrm{l}$ of plasma spiked with 10 pmol internal standard 18:1-D7S1P (Avanti Polar Lipids, Alabaster, AL, USA). Analysis of S1P species included the quantification of 16:1-S1P, 17:1-S1P, 18:1-S1P and 18:0-S1P lipids.

\subsection{Quantification of sphingoid bases}

The profile of 11 plasma sphingoid bases was analyzed by LC-MS as described earlier [23]. d7-sphingosine (D7SO) and d7sphinganine (D7SA) (200 pmol each) were used as internal standards (Avanti Polar Lipids, Alabaster, AL, USA).

\subsection{Statistical analysis}

Statistical analysis was performed using SPSS, version 19 (IBM Corporation, Somers NY, USA). Normality of the data was determined by using the Kolmogorov-Smirnov test. For variables which did not show a normal distribution, univariate statistics was done using Kruskal-Wallis and the Mann-Whitney U tests. Categorical variables were compared using the Chi-square test. Spearman rank tests were used to describe the correlations of plasma glycerophospholipids and sphingolipids with total cholesterol, LDL-C and HDL-C levels. The $p$ values were adjusted for multiple comparisons by applying the Benjamini-Hochberg procedure [24]. Adjusted (adj.) $p$ values of $<0.05$ were considered statistically significant.

\section{Results}

\subsection{Patient characteristics}

Here we compared the plasma lipid profile between healthy subjects and patients with stable CAD $(n=18)$ or AMI $(n=17)$. The age of the patients ranged between 43 and 77 years. On average, healthy subjects $(56.4 \pm 7.5$ years $)$ were ten and patients with CAD $(61.3 \pm 8.3$ years $)$ five years younger than patients with AMI $(66.8 \pm 10.0$ years). Features of acute myocardial infarction, including elevated levels of troponin T, NT-proBNP, creatine kinase and CK-MB were higher in the AMI group (Table 1). Prevalence of smoking was significantly higher in CAD and AMI group. Average BMI was higher in patients with CAD compared to healthy subjects. LDL-C and total cholesterol levels were lower in patients with stable CAD and AMI due to statin treatment.

\subsection{Plasma lipidome - alterations in patients with CAD and AMI}

Sixty-five lipid species were analyzed in this study. As statin use was significantly higher in the CAD and AMI group (Table 1) we first tested for an influence of statins on the analyzed lipids species by comparing CAD patients with and without statin treatment (Table 2). No significant differences for the analyzed glycerophospholipid and sphingolipid species were observed between the two groups.

Next we compared the same lipid profile between healthy subjects and patients with CAD and AMI. Univariate statistics revealed significant changes in the plasma lipid profile for patients with stable CAD and AMI compared to healthy individuals (Table 3). The total levels of PC, LPC, PE, SM and Cer/HexCer lipids were generally lower in patients with stable CAD and AMI compared to healthy subjects (Table 3). Total Cer and HexCer were further reduced in patients with AMI compared to the CAD group. Comparing the individual lipid species we observed differences in 45 molecular species (19 PCs, 4 LPCs, 5 PEs, 12 SMs and 5 Cers/ HexCers) for the CAD and differences in 42 lipid species (15 PCs, 2 LPCs, 9 PEs, 11 SMs and 5 Cers/HexCers) for the AMI group in comparison to healthy subjects (Table 3 ).

Sphingoid bases are the shared structural component for all sphingolipids. C18 sphingosine (d18:1) is typically the most abundant sphingoid base in plasma although a wide range of other sphingoid bases can be found too. For sphingoid base analysis, total sphingolipids were extracted from plasma samples and hydrolyzed to remove the conjugated $\mathrm{N}$-acyl chain and head groups. It is important to note that the here reported sphingoid base concentrations refer to the total levels of a given sphingoid base in plasma irrespective of the sphingolipid specie where it was originally contained (e.g Cer, hexCer, SM etc). In the CAD and AMI group, we observe lower plasma levels for $\mathrm{C}_{17} \mathrm{SO}, \mathrm{C}_{18} \mathrm{SO}, \mathrm{C}_{18} \mathrm{SA}$ and $\mathrm{C}_{18}$ SAdiene

Table 1

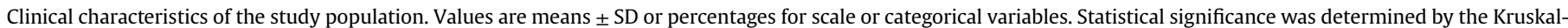

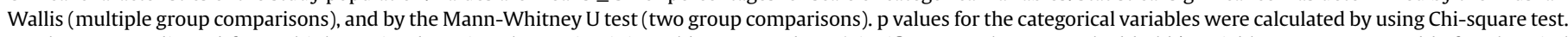

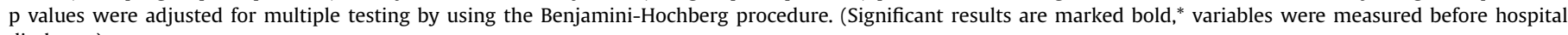
discharge).

\begin{tabular}{|c|c|c|c|c|c|c|c|}
\hline \multirow[t]{2}{*}{ Characteristics } & \multirow{2}{*}{$\frac{\text { Healthy subjects }(\mathrm{n}=14)}{\text { Mean } \pm \mathrm{SD}}$} & \multirow{2}{*}{$\frac{\mathrm{CAD}(\mathrm{n}=18)}{\text { Mean } \pm \mathrm{SD}}$} & \multirow{2}{*}{$\frac{\text { AMI }(\mathrm{n}=17)}{\text { Mean } \pm \mathrm{SD}}$} & \multirow[t]{2}{*}{ Kruskal-Wallis, adj. $p$ Values } & \multicolumn{3}{|c|}{ Mann-Whitney $\mathrm{U}$, adj. $p$ Values } \\
\hline & & & & & $\mathrm{H}$ vs CAD & H vs ACS & CAD vs ACS \\
\hline Age (years) & $56.4 \pm 7.5$ & $61.3 \pm 8.3$ & $66.8 \pm 10.0$ & 0.005 & 0.164 & 0.004 & 0.084 \\
\hline Gender, female (\%) & 36 & 22 & 18 & 0.488 & 0.492 & 0.347 & 0.784 \\
\hline Smoking (\%) & 0 & 75 & 56 & 0.001 & $<0.001$ & 0.04 & 0.352 \\
\hline Statin treatment (\%) & 0 & 100 & 100 & $<0.001$ & $<0.001$ & $<0.001$ & 0.649 \\
\hline BMI (kg/m2) & $24.5 \pm 3.48$ & $27.85 \pm 3.52$ & $26.45 \pm 3.15$ & 0.03 & 0.035 & 0.084 & 0.22 \\
\hline Systolic blood pressure (mmHg) & $119.36 \pm 13.99$ & $129.89 \pm 16.52$ & $128.59 \pm 19.11$ & 0.248 & 0.163 & 0.232 & 0.92 \\
\hline Diastolic blood pressure (mmHg) & $71.36 \pm 9.79$ & $76.50 \pm 10.76$ & $76.06 \pm 11.27$ & 0.267 & 0.22 & 0.215 & 0.981 \\
\hline Glucose (mmol/l) & $5.18 \pm 0.44$ & $5.43 \pm 0.56$ & $6.32 \pm 0.98$ & 0.005 & 0.362 & 0.006 & 0.018 \\
\hline Total cholesterol (mmol/l) & $5.78 \pm 0.97$ & $4.05 \pm 0.89$ & $3.84 \pm 1.00$ & $<0.001$ & $<\mathbf{0 . 0 0 1}$ & $<\mathbf{0 . 0 0 1}$ & 0.448 \\
\hline LDL-C $(\mathrm{mmol} / \mathrm{l})$ & $3.53 \pm 0.83$ & $2.39 \pm 0.76$ & $2.28 \pm 0.92$ & 0.001 & 0.004 & 0.004 & 0.609 \\
\hline HDL-C (mmol/l) & $1.83 \pm 0.42$ & $1.18 \pm 0.32$ & $1.22 \pm 0.28$ & $<0.001$ & $<0.001$ & 0.001 & 0.618 \\
\hline Triglycerides (mmol/l) & $0.98 \pm 0.43$ & $1.07 \pm 0.46$ & $0.75 \pm 0.53$ & 0.099 & 0.738 & 0.132 & 0.081 \\
\hline NT-proBNP (ng/L) & $41.21 \pm 35.36$ & $140.22 \pm 108.47$ & $817.88 \pm 1013.81$ & $<0.001$ & 0.006 & $<0.001$ & 0.026 \\
\hline Creatine kinase $(\mu \mathrm{g} / \mathrm{l})$ & $136.18 \pm 76.96$ & $119.65 \pm 42.47$ & $1304.76 \pm 828.56$ & $<0.001$ & 0.981 & $<0.001$ & $<0.001$ \\
\hline CK-MB $(\mu \mathrm{g} / \mathrm{l})^{*}$ & $11.60 \pm 3.47$ & $10.86 \pm 5.41$ & $141.49 \pm 103.43$ & $<0.001$ & 0.558 & $<0.001$ & $<0.001$ \\
\hline Troponin T (ug/l)* & $0.01 \pm 0$ & $0.03 \pm 0.03$ & $2.65 \pm 1.89$ & $<0.001$ & 0.013 & $<0.001$ & $<\mathbf{0 . 0 0 1}$ \\
\hline
\end{tabular}

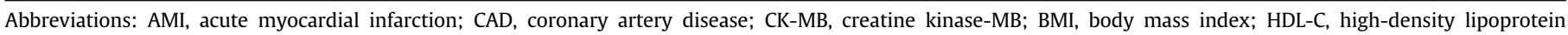
cholesterol; LDL-C, low-density lipoprotein cholesterol; NT-proBNP, N-terminal pro-B-type natriuretic peptide. 
Table 2

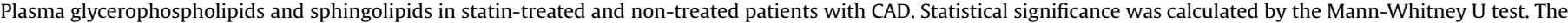

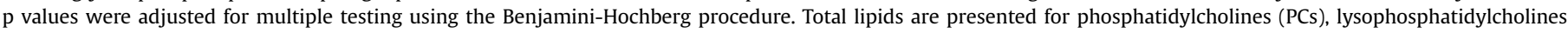

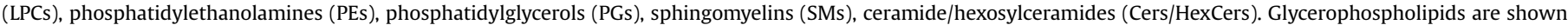

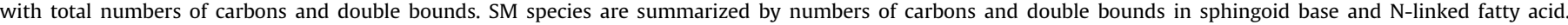

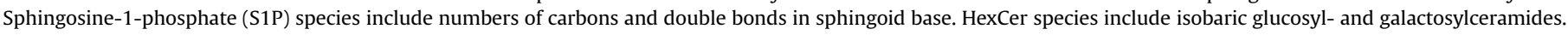
(n.d. - not detected).

\begin{tabular}{|c|c|c|c|}
\hline \multirow[t]{2}{*}{ Lipids $(\mu \mathrm{M})$} & \multirow{2}{*}{$\frac{\text { CAD statin-treated }(\mathrm{n}=10)}{\text { Median(Min; Max })}$} & \multirow{2}{*}{$\frac{\text { CAD statin-untreated }(\mathrm{n}=10)}{\text { Median(Min; Max })}$} & \multirow{2}{*}{$\begin{array}{l}\text { Mann-Whitney } \mathrm{U} \text {, adj. } p \text { values } \\
\text { CAD statin-treated vs } \\
\text { CAD statin-untreated }\end{array}$} \\
\hline & & & \\
\hline Total PC & $795.3(614.8 ; 895.6)$ & $823.6(692.2 ; 1195.8)$ & 0.649 \\
\hline PC32:0 & $5.6(3.1 ; 7.8)$ & $6.8(4.6 ; 9.5)$ & 0.406 \\
\hline PC32:1 & $8.5(3 ; 15.5)$ & $11.6(3.6 ; 15.4)$ & 0.649 \\
\hline PC33:1 & $5.3(2.4 ; 9)$ & $6.7(4.3 ; 9.3)$ & 0.402 \\
\hline PC33:2 & $5.4(3.3 ; 12.3)$ & $7(3.7 ; 10.5)$ & 0.402 \\
\hline PC33:3 & $2.8(1.4 ; 5.9)$ & $3.5(2.5 ; 7.8)$ & 0.48 \\
\hline PC $34: 0$ & $7.5(4.4 ; 11.7)$ & $9.1(5 ; 11.6)$ & 0.553 \\
\hline PC34:1 & $87.4(50 ; 126.5)$ & $112.9(77.4 ; 152.8)$ & 0.402 \\
\hline PC34:2 & $152.2(88 ; 222.1)$ & $174.5(115.6 ; 297.4)$ & 0.649 \\
\hline PC34:3 & $6.3(4.3 ; 9)$ & $7.3(3.9 ; 10.6)$ & 0.498 \\
\hline PC35:1 & $2.7(1 ; 3.5)$ & $3.8(1.9 ; 5.5)$ & 0.402 \\
\hline PC35:2 & $5.8(3.5 ; 6.3)$ & $6.9(4.7 ; 11.2)$ & 0.465 \\
\hline PC35:3 & $4.4(2.3 ; 8)$ & $5.6(3.9 ; 9.7)$ & 0.429 \\
\hline PC35:4 & $11.6(8.9 ; 17.9)$ & $14.9(8.6 ; 19.3)$ & 0.48 \\
\hline PC35:5 & $7.8(4.8 ; 15.3)$ & $9.3(6.8 ; 12.9)$ & 0.649 \\
\hline PC36:0 & $4(2.8 ; 5.7)$ & $3.9(1.8 ; 6.6)$ & 0.837 \\
\hline PC36:1 & $22.6(16.8 ; 31.2)$ & $31.1(18.9 ; 42.7)$ & 0.402 \\
\hline PC36:2 & $81.8(56.3 ; 115.2)$ & $86.4(67.2 ; 144.3)$ & 0.702 \\
\hline PC36:3 & $53(41.6 ; 68.1)$ & $54.9(38 ; 77.5)$ & 0.869 \\
\hline PC36:4 & $81.8(47.4 ; 103.9)$ & $74.5(53.9 ; 120.8)$ & 0.869 \\
\hline PC36:5 & $8.6(2 ; 19.8)$ & $9.9(6.2 ; 15.6)$ & 0.759 \\
\hline PC37:4 & $10.3(7.2 ; 15.9)$ & $11.6(7.3 ; 14.2)$ & 0.649 \\
\hline PC37:5 & $12.5(7.8 ; 16.4)$ & $15.2(9.3 ; 21.6)$ & 0.465 \\
\hline PC37:6 & $5.3(4.1 ; 9)$ & $6.7(4.6 ; 9.9)$ & 0.406 \\
\hline PC38:3 & $27.7(18.9 ; 37.1)$ & $24.1(20.4 ; 43.3)$ & 0.9 \\
\hline PC $38: 4$ & $65.1(28.3 ; 75.7)$ & $55.8(37.7 ; 101.3)$ & 0.735 \\
\hline PC38:5 & $23.3(12.3 ; 30)$ & $21.8(17.3 ; 27)$ & 0.735 \\
\hline PC38:6 & $24.9(10.4 ; 34.5)$ & $24(15.5 ; 31.5)$ & 0.9 \\
\hline PC40:5 & $6.1(4.3 ; 7.4)$ & $6.1(4.5 ; 9.6)$ & 0.848 \\
\hline PC40:6 & $10.6(5 ; 14.1)$ & $10.3(5.7 ; 15.7)$ & 0.869 \\
\hline Total LPC & $123.6(80.5 ; 154.7)$ & $129.3(88.7 ; 179.2)$ & 0.735 \\
\hline LPC16:0 & $67.4(44.8 ; 77.5)$ & $75.2(53 ; 96.7)$ & 0.48 \\
\hline LPC18:0 & $21.3(14.8 ; 27.7)$ & $25.8(12 ; 34)$ & 0.649 \\
\hline LPC18:1 & $14.7(8.8 ; 20.4)$ & $14(9 ; 26.2)$ & 0.702 \\
\hline LPC18:2 & $20.6(8.8 ; 37.9)$ & $15.1(9.1 ; 32.3)$ & 0.649 \\
\hline Total PE & $14.2(6.3 ; 40.2)$ & $16.3(8.2 ; 70)$ & 0.759 \\
\hline PE34:2 & $0.9(0.2 ; 3.3)$ & $1.6(0.3 ; 5.5)$ & 0.649 \\
\hline PE35:2 & $0.1(0.03 ; 0.3)$ & $0.3(0.1 ; 0.6)$ & 0.402 \\
\hline PE36:4 & $1.3(0.8 ; 3.4)$ & $1.7(0.5 ; 6.2)$ & 0.837 \\
\hline PE36:3 & $0.8(0.1 ; 1.7)$ & $0.9(0.2 ; 4.5)$ & 0.759 \\
\hline PE36:2 & $2.8(0.7 ; 8.7)$ & $3.5(0.6 ; 20.6)$ & 0.759 \\
\hline PE36:1 & $0.6(0.1 ; 2.5)$ & $0.8(0.1 ; 5.3)$ & 0.56 \\
\hline PE37:5 & $0.4(0.03 ; 0.5)$ & $0.8(0.2 ; 1.3)$ & 0.402 \\
\hline PE37:4 & $0.2(0.1 ; 0.6)$ & $0.2(0.1 ; 0.5)$ & 0.848 \\
\hline PE38:6 & $2(0.5 ; 7.3)$ & $2.2(0.9 ; 7.4)$ & 0.702 \\
\hline PE38:5 & $1(0.6 ; 2.5)$ & $1.3(0.5 ; 3.2)$ & 0.804 \\
\hline PE38:4 & $3.9(1.9 ; 10.4)$ & $4.4(2.1 ; 15.6)$ & 0.804 \\
\hline Total PG & $0.4(0.2 ; 0.7)$ & $0.4(0.2 ; 1.7)$ & 0.94 \\
\hline Total PA & n.d. & n.d. & - \\
\hline Total SM & $502.6(283.2 ; 628)$ & $560.4(428.8 ; 702)$ & 0.465 \\
\hline SM32:1 & $11.1(5.5 ; 18)$ & $14.6(12 ; 17.7)$ & 0.402 \\
\hline SM34:0 & $4(1.1 ; 5)$ & $4.4(1.5 ; 6.1)$ & 0.694 \\
\hline SM34:1 & $100.3(62.1 ; 136)$ & $115.8(86.2 ; 161.1)$ & 0.402 \\
\hline SM34:2 & $14.6(8.5 ; 22.8)$ & $16.2(12.5 ; 28.3)$ & 0.498 \\
\hline SM36:1 & $29.9(15.6 ; 42)$ & $26.3(22.9 ; 46.6)$ & 0.94 \\
\hline SM36:2 & $10.3(7.1 ; 14.3)$ & $11.4(8.1 ; 16.8)$ & 0.735 \\
\hline SM38:1 & $26.7(15 ; 32.1)$ & $29.4(21.5 ; 36.6)$ & 0.465 \\
\hline SM39:1 & $9.9(5.2 ; 16.7)$ & $10.9(6.9 ; 15)$ & 0.702 \\
\hline SM39:2 & $1.3(0.8 ; 2.3)$ & $1.5(0.7 ; 1.9)$ & 0.694 \\
\hline SM40: 1 & $53.7(28.3 ; 66)$ & $67.5(42.8 ; 95)$ & 0.406 \\
\hline SM40:2 & $31.9(19 ; 39.5)$ & $38.9(24.7 ; 49.5)$ & 0.402 \\
\hline SM41:1 & $26.2(11.1 ; 38)$ & $34.7(23.7 ; 42.7)$ & 0.406 \\
\hline SM41:2 & $17.1(8.8 ; 25.6)$ & $21.9(14.3 ; 31.2)$ & 0.618 \\
\hline SM42:1 & $43(20.7 ; 52.3)$ & $48.7(33.7 ; 75.6)$ & 0.649 \\
\hline SM42:2 & $74.3(53.1 ; 113.7)$ & $88.1(68.1 ; 123.5)$ & 0.56 \\
\hline
\end{tabular}


Table 2 (continued)

\begin{tabular}{|c|c|c|c|}
\hline \multirow[t]{2}{*}{ Lipids $(\mu \mathrm{M})$} & CAD statin-treated $(\mathrm{n}=10)$ & CAD statin-untreated $(\mathrm{n}=10)$ & Mann-Whitney $U$, adj. $p$ values \\
\hline & Median(Min; Max) & Median(Min; Max) & $\begin{array}{l}\text { CAD statin-treated vs } \\
\text { CAD statin-untreated }\end{array}$ \\
\hline SM42:3 & $28.9(21.3 ; 39.5)$ & $34(19.9 ; 48.5)$ & 0.702 \\
\hline Total Cer/HexCer & $7.6(4.6 ; 13.4)$ & $9.4(6.5 ; 21.5)$ & 0.465 \\
\hline $\operatorname{Cer}(\mathrm{d} 18: 1 / 24: 0)$ & $2.3(1.5 ; 3.5)$ & $2.6(1.4 ; 5.4)$ & 0.837 \\
\hline HexCer(d18:1/22:0) & $1.4(0.8 ; 2.1)$ & $1.3(0.5 ; 5.3)$ & 0.848 \\
\hline HexCer(d18:1/23:0) & $0.6(0.1 ; 1.5)$ & $1.4(0.6 ; 2.4)$ & 0.402 \\
\hline HexCer(d18:1/24:0) & $2(1.1 ; 3.7)$ & $2.8(1.4 ; 6.5)$ & 0.429 \\
\hline HexCer(d18:1/24:1) & $1.2(0.6 ; 2.6)$ & $1.7(0.7 ; 2.9)$ & 0.649 \\
\hline \multicolumn{4}{|l|}{ S1P species } \\
\hline 16:1-S1P & $0.1(0.07 ; 0.15)$ & $0.11(0.07 ; 0.13)$ & 0.649 \\
\hline 17:1-S1P & n.d. & n.d. & - \\
\hline 18:1-S1P & $0.45(0.39 ; 0.64)$ & $0.55(0.51 ; 0.78)$ & 0.402 \\
\hline 18:0-S1P & $0.08(0.04 ; 0.15)$ & $0.06(0.03 ; 0.1)$ & 0.649 \\
\hline \multicolumn{4}{|c|}{ Sphingoid bases of sphingolipids } \\
\hline C16SO & $21.96(10.47 ; 29.5)$ & 23.45(13.69; 27.98) & 0.735 \\
\hline C16SA & $0.56(0.25 ; 0.99)$ & $0.55(0.27 ; 0.8)$ & 0.9 \\
\hline C17SO & $9.09(4.43 ; 10.95)$ & $10.31(6.92 ; 14.97)$ & 0.406 \\
\hline C18SO & $89.08(57.3 ; 122.08)$ & $112.52(79.23 ; 140.89)$ & 0.498 \\
\hline C18SA & $3.29(1.4 ; 5.36)$ & $3.35(1.85 ; 6.49)$ & 0.848 \\
\hline C18SAdiene & $34.81(24.08 ; 46.54)$ & $41.76(25.29 ; 51.8)$ & 0.402 \\
\hline C19SO & $3.56(1.71 ; 6.75)$ & $3.34(1.73 ; 4.68)$ & 0.869 \\
\hline $\mathrm{C} 20 \mathrm{SO}$ & $0.23(0.2 ; 0.37)$ & $0.22(0.18 ; 0.34)$ & 0.735 \\
\hline C20SA & $0.04(0.02 ; 0.06)$ & $0.04(0.02 ; 0.06)$ & 0.848 \\
\hline doxSO & $0.18(0.09 ; 0.39)$ & $0.18(0.04 ; 0.4)$ & 0.735 \\
\hline doxSA & $0.09(0.04 ; 0.18)$ & $0.09(0.02 ; 0.17)$ & 0.735 \\
\hline
\end{tabular}

based sphingolipids compared to healthy subjects but no difference between patients with CAD and AMI (Table 3). Plasma levels of C16SO and C16SA were significantly lower in patients with AMI compared to healthy controls (Table 3). 1-Deoxysphingoid bases were not significantly altered between the groups.

The analysis of S1P species showed the presence of canonical 18:1-S1P but also of other S1P species, including 16:1-S1P, 17:1-S1P and 18:0-S1P. Canonical (18:1) S1P was lower in patients with AMI compared to healthy individuals (Table 3) whereas an atypical 16:1-S1P was lower in both groups (Table 3). Also 17:1-S1P was detectable but levels were below the limit of quantification $(0.04 \mu \mathrm{M})$.

For further analysis, lipid and clinical data were arranged as volcano plots, showing the relative difference ( $\mathrm{x}$-axes) versus significance (y-axes). The analyzed lipid species were divided into four groups: 1. glycerophospholipids including even-chain PCs, PEs, LPCs and PGs; 2. glycerophospholipids including odd-chain PCs; 3. sphingolipids including SMs, Cers/HexCers, S1Ps and sphingoid bases and 4. clinical measures. Comparisons of healthy subjects vs. patients with CAD are shown in Fig. 1A, comparisons of healthy subjects vs. patients with AMI in Fig. $1 \mathrm{~B}$, and comparisons of CAD vs. AMI in Fig. 1C. The analysis revealed four odd-chain PC species (PC33:1, PC33:2, PC33:3 and PC35:3) to be the most significantly lowered species in patients with CAD compared to healthy subjects (Fig. 1A). The same odd-chain PC species were found to be lowered in the AMI group (Fig. 1B). Comparing CAD and AMI did not reveal differences in the lipid species but differences in some clinical variables including troponin, creatine kinase and CK-MB (Fig. 1C).

\subsection{Structural analysis identifies odd-chain phosphatidylcholines as PC plasmalogens}

The four identified odd-chain PC species (PC33:1, PC33:2, PC33:3 and PC35:3) could be either PCs with an odd number of carbons or PC plasmalogens with a vinyl-ether bond at the sn-1 position of the glycerol backbone. Comparing the MS/MS fragmentation pattern of these species to a commercial standard we already identified $\mathrm{PC} 35: 3$ as a $\mathrm{PC}(\mathrm{P}-18: 0 / 18: 2)$ plasmalogen in a different study [13]. A similar structural analysis of the PC33:1, PC33:2 and PC33:3 species confirmed them as plasmalogens PC(P-18:0/16:0), PC(P-16:0/18:1) and PC(P-16:0/18:2), respectively. The identity of other odd-chain PCs species in the dataset was not further investigated.

\subsection{Plasma glycerophospholipids and sphingolipids correlate with cholesterol levels}

Most of the identified glycerophospholipids and sphingolipids showed a significant correlation with total cholesterol and LDL-C (Fig. 2). Correlation with HDL-C was also seen for most species but less pronounced. Among all glycerophospholipid and sphingolipid species, PC33:1, PC33:2, PC33:3 and PC35:3 showed the strongest correlation with HDL-C $(r=0.81, r=0.71, r=0.75$ and $r=0.68$, respectively). Furthermore, total cholesterol and LDL-C correlated strongly with the sphingoid bases C18SO ( $r=0.93$ and $r=0.86$, respectively) and C17SO $(r=0.85$ and $r=0.81$, respectively). Saturated LPC species correlated more with total cholesterol and LDL-C than with HDL-C.

\subsection{Glycerophospholipid and sphingolipid levels in the lipoprotein fractions}

Because of the strong correlations between the individual lipoproteins and plasma glycerophospholipids and sphingolipid species, we analyzed the distribution of these lipids across the major lipoprotein fractions. Lipoproteins were isolated from plasma of three healthy volunteers and the lipid profile analyzed in Chylomicrons/VLDL, LDL, HDL and in the lipoprotein-free fraction (LFF). Species for PC, SM and LPC were detected in all plasma lipoprotein fractions. PE and Cer/HexCer species were also present in lipoproteins, but at levels below the limit of quantification. Neither PG nor PA lipids were identified in the lipoprotein or LFF fractions.

The relative percentages of total PC, SM and LPC lipids in the individual lipoprotein fractions are shown in Fig. 3A-C. The percentages of the individual PC, SM and LPC species relative to the total concentration of a given species in all lipoprotein fractions is 
Table 3

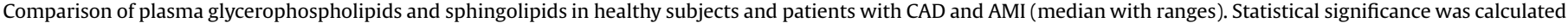

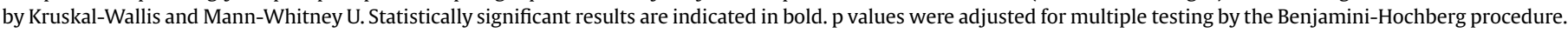
(AMI acute myocardial infarction; CAD, coronary artery disease; n.d., not detected).

\begin{tabular}{|c|c|c|c|c|c|c|c|}
\hline \multirow[t]{2}{*}{ Lipids, $\mu \mathrm{mol} / \mathrm{l}$} & \multirow{2}{*}{$\frac{\text { Healthy subjects }(\mathrm{n}=14)}{\text { Median(Min; Max })}$} & \multirow{2}{*}{$\frac{\operatorname{CAD}(\mathrm{n}=18)}{\operatorname{Median}(\operatorname{Min} ; \operatorname{Max})}$} & \multirow{2}{*}{$\frac{\operatorname{AMI}(\mathrm{n}=17)}{\operatorname{Median}(\operatorname{Min} ; \operatorname{Max})}$} & \multirow[t]{2}{*}{ Kruskal-Wallis, adj. $p$ values } & \multicolumn{3}{|c|}{ Mann-Whitney $\mathrm{U}$, adj. $p$ values } \\
\hline & & & & & $\mathrm{H}$ vs CAD & $\mathrm{H}$ vs ACS & CAD vs ACS \\
\hline Total PC & 829.3(692.3; 974.1) & $725.6(522.7 ; 793.4)$ & $670.4(595.9 ; 868.8)$ & $<0.001$ & 0.002 & 0.001 & 0.38 \\
\hline PC32:0 & $6.8(5 ; 9.3)$ & $5(3.3 ; 6.8)$ & $5.6(3.5 ; 7.9)$ & 0.002 & 0.002 & 0.02 & 0.314 \\
\hline PC32:1 & $9(4.9 ; 18.4)$ & $6.2(3.2 ; 12)$ & $7.3(4.2 ; 16.6)$ & 0.094 & 0.05 & 0.2 & 0.697 \\
\hline PC33:1 & $7.7(7 ; 11.7)$ & $5.5(2.5 ; 6.7)$ & $5.8(3.9 ; 7.9)$ & $<0.001$ & $<0.001$ & 0.001 & 0.584 \\
\hline PC33:2 & $10.1(6.7 ; 16)$ & $5.9(2.6 ; 7.3)$ & $6.4(4.3 ; 8.8)$ & $<0.001$ & $<0.001$ & 0.001 & 0.314 \\
\hline PC33:3 & $5.6(3.6 ; 10.7)$ & $2.8(1.7 ; 4.2)$ & $3.2(1.6 ; 4.7)$ & $<0.001$ & $<0.001$ & $<0.001$ & 0.584 \\
\hline PC34:0 & $9.3(6.3 ; 12.7)$ & $7.1(4.3 ; 10.5)$ & $7.4(5.4 ; 9.2)$ & 0.004 & 0.015 & 0.002 & 0.877 \\
\hline PC34:1 & $92.6(74.9 ; 122.2)$ & $76.2(42 ; 101.3)$ & $81.7(51.7 ; 101.2)$ & 0.043 & 0.026 & 0.108 & 0.681 \\
\hline PC34:2 & $187.8(156.6 ; 226.7)$ & $135.9(72.2 ; 181.7)$ & $129.5(98 ; 150.1)$ & $<0.001$ & $<0.001$ & $<0.001$ & 0.471 \\
\hline PC $34: 3$ & $8.7(5.1 ; 9.7)$ & $5.5(3.6 ; 8.1)$ & $4.5(3.2 ; 6.8)$ & $<0.001$ & 0.002 & 0.001 & 0.391 \\
\hline PC35:1 & $10.3(8 ; 20.6)$ & $7.4(3.5 ; 13.1)$ & $7.5(5.8 ; 12.3)$ & 0.004 & 0.006 & 0.006 & 0.863 \\
\hline PC35:2 & $8.1(7 ; 11.4)$ & $6.3(2.9 ; 8.8)$ & $5.9(4.2 ; 9.3)$ & $<0.001$ & 0.001 & 0.002 & 0.681 \\
\hline PC35:3 & $7.5(6 ; 11)$ & $5.1(2.3 ; 7.4)$ & $5.1(2.7 ; 6.9)$ & $<0.001$ & $<0.001$ & $<0.001$ & 0.932 \\
\hline PC35:4 & $15.4(8.7 ; 22.6)$ & $13(7.4 ; 15.8)$ & $13.1(7.6 ; 20.7)$ & 0.031 & 0.015 & 0.116 & 0.644 \\
\hline PC35:5 & $11.9(6.1 ; 21)$ & $8.4(4.4 ; 11.2)$ & $9.2(4.7 ; 17.6)$ & 0.041 & 0.024 & 0.148 & 0.482 \\
\hline PC36:0 & $5.3(3.8 ; 7.7)$ & $4(2.6 ; 5.4)$ & $4(2.4 ; 6.7)$ & 0.005 & 0.003 & 0.022 & 0.877 \\
\hline PC36:1 & $32.7(23.9 ; 38.3)$ & $22.7(13.4 ; 30.5)$ & $22.7(17.1 ; 33.3)$ & $<0.001$ & $<0.001$ & 0.002 & 0.681 \\
\hline PC36:2 & $106.7(77.7 ; 124.3)$ & $78.6(51.9 ; 112.1)$ & $72.1(52.8 ; 87.3)$ & $<0.001$ & 0.001 & $<0.001$ & 0.509 \\
\hline PC36:3 & $48.7(44 ; 68.8)$ & $44.7(29.7 ; 57.6)$ & $40.7(29.8 ; 47.2)$ & 0.001 & 0.09 & 0.001 & 0.175 \\
\hline PC36:4 & $70(39.4 ; 84.3)$ & $73.7(44 ; 102)$ & $74.4(47.4 ; 89.1)$ & 0.682 & 0.506 & 0.595 & 0.932 \\
\hline PC36:5 & $8.8(5.8 ; 21.7)$ & $8.8(1.3 ; 14.9)$ & $9.2(2 ; 22.7)$ & 0.725 & 0.491 & 0.877 & 0.768 \\
\hline PC37:4 & $12.7(8.3 ; 17)$ & $11.4(6 ; 14.1)$ & $10.8(7.3 ; 15.3)$ & 0.226 & 0.235 & 0.159 & 0.863 \\
\hline PC37:5 & $17.3(11 ; 23.1)$ & $13.5(7.7 ; 18.6)$ & $14.5(9 ; 22.5)$ & 0.026 & 0.018 & 0.085 & 0.509 \\
\hline PC37:6 & $8.6(5.9 ; 14.9)$ & $6.2(3.4 ; 8)$ & $7(3.3 ; 11.6)$ & 0.003 & 0.002 & 0.028 & 0.286 \\
\hline PC38:3 & $21.6(11.2 ; 31.9)$ & $23.5(14.4 ; 38.5)$ & $19.8(10.9 ; 28.6)$ & 0.157 & 0.506 & 0.386 & 0.091 \\
\hline PC38:4 & 49.5(28.9; 70.1) & $60.7(41.5 ; 77.5)$ & $60.6(38.4 ; 72.8)$ & 0.091 & 0.047 & 0.29 & 0.482 \\
\hline PC38:5 & $19.3(11.8 ; 26.7)$ & $18.3(8.2 ; 28.1)$ & $18.9(12.2 ; 27.7)$ & 0.979 & 0.95 & 0.877 & 0.863 \\
\hline РС38:6 & $17(12.7 ; 34.3)$ & $20.7(9.3 ; 32.4)$ & $18.7(11.1 ; 34.6)$ & 0.259 & 0.148 & 0.815 & 0.38 \\
\hline PC40:5 & $5.3(3.3 ; 7.1)$ & $5.9(3.5 ; 8.8)$ & $5(2.8 ; 9.2)$ & 0.387 & 0.34 & 0.904 & 0.343 \\
\hline PC40:6 & $7.7(5.1 ; 14.1)$ & $10(5.5 ; 14.2)$ & $8.5(5.7 ; 16.1)$ & 0.201 & 0.104 & 0.506 & 0.482 \\
\hline Total LPC & $179.2(156.8 ; 245.2)$ & $136.3(105.3 ; 181.5)$ & $136.6(110.5 ; 199.9)$ & $<0.001$ & 0.001 & 0.004 & 0.623 \\
\hline LPC16:0 & $90.8(77.4 ; 121.1)$ & $72.5(52.3 ; 95.9)$ & $64.1(49.6 ; 104.2)$ & $<0.001$ & 0.001 & 0.001 & 0.235 \\
\hline LPC18:0 & $30.7(26.4 ; 46.7)$ & $25.6(20 ; 34.1)$ & $22(15.7 ; 33.3)$ & 0.001 & 0.006 & 0.002 & 0.098 \\
\hline LPC18:1 & $21.3(15.4 ; 28.5)$ & $15.6(10.6 ; 21.8)$ & $21.2(9.1 ; 27.6)$ & 0.004 & 0.004 & 0.572 & 0.022 \\
\hline LPC18:2 & $32.5(25.6 ; 50.6)$ & $19.5(9.6 ; 50)$ & $32.1(11.6 ; 71.6)$ & 0.005 & 0.005 & 0.67 & 0.024 \\
\hline Total PE & $16.3(9.9 ; 26)$ & $11.2(3.4 ; 23.4)$ & $9.4(5 ; 17.6)$ & 0.003 & 0.028 & 0.003 & 0.26 \\
\hline PE34:2 & $1.7(0.9 ; 3.2)$ & $0.7(0.1 ; 2.3)$ & $0.6(0.3 ; 1.8)$ & 0.001 & 0.003 & 0.001 & 0.697 \\
\hline PE35:2 & $0.3(0.1 ; 0.5)$ & $0.1(0.03 ; 0.3)$ & $0.1(0.01 ; 0.2)$ & $<0.001$ & 0.002 & 0.001 & 0.768 \\
\hline PE36:4 & $1.4(0.8 ; 2.7)$ & $1(0.3 ; 2.3)$ & $0.9(0.4 ; 2)$ & 0.07 & 0.11 & 0.049 & 0.721 \\
\hline PE36:3 & $1.1(0.5 ; 3.1)$ & $0.6(0.02 ; 1.3)$ & $0.5(0.2 ; 0.8)$ & 0.001 & 0.006 & 0.002 & 0.605 \\
\hline PE36:2 & $4(2.1 ; 8.5)$ & $1.9(0.4 ; 5.1)$ & $1.6(0.5 ; 3.4)$ & $<0.001$ & 0.005 & 0.001 & 0.248 \\
\hline PE36: 1 & $0.7(0.2 ; 2)$ & $0.5(0.05 ; 1.1)$ & $0.4(0.1 ; 1)$ & 0.041 & 0.065 & 0.049 & 0.391 \\
\hline PE37:5 & $0.7(0.4 ; 1.1)$ & $0.4(0.1 ; 0.7)$ & $0.4(0.2 ; 1)$ & 0.004 & 0.003 & 0.015 & 0.79 \\
\hline PE37:4 & $0.4(0.2 ; 0.8)$ & $0.3(0.02 ; 0.6)$ & $0.2(0.1 ; 0.4)$ & 0.014 & 0.069 & 0.006 & 0.623 \\
\hline PE38:6 & $1.6(0.5 ; 3.7)$ & $1.6(0.4 ; 4)$ & $1.1(0.5 ; 2.9)$ & 0.401 & 0.857 & 0.276 & 0.449 \\
\hline PE38:5 & $1.1(0.4 ; 2.6)$ & $0.8(0.3 ; 2)$ & $0.8(0.4 ; 1.8)$ & 0.068 & 0.069 & 0.065 & 0.932 \\
\hline PE38:4 & $2.9(1.9 ; 6.3)$ & $2.9(1 ; 5.5)$ & $2.3(1.3 ; 4.9)$ & 0.055 & 0.811 & 0.049 & 0.079 \\
\hline Total PG & $0.3(0.1 ; 0.8)$ & $0.3(0.1 ; 0.7)$ & $0.2(0.1 ; 0.6)$ & 0.099 & 0.543 & 0.108 & 0.117 \\
\hline Total PA & n.d. & n.d. & n.d. & - & - & - & - \\
\hline Total SM & $462.9(359.6 ; 600.4)$ & $385.4(262.7 ; 547.9)$ & $355.4(275.6 ; 475.7)$ & 0.002 & 0.007 & 0.002 & 0.523 \\
\hline SM32:1 & $16.1(12.2 ; 22.6)$ & $10.7(5.3 ; 17.8)$ & $10.7(5.3 ; 16.4)$ & 0.001 & 0.006 & 0.001 & 0.972 \\
\hline SM34:0 & $4.8(3.1 ; 6.1)$ & $2.9(0.3 ; 4.5)$ & $2.7(1.1 ; 4.2)$ & 0.001 & 0.003 & 0.002 & 0.863 \\
\hline SM34:1 & $123.6(88.3 ; 169.2)$ & $99.3(63 ; 131)$ & $90.6(63.8 ; 127.6)$ & $<0.001$ & 0.002 & 0.001 & 0.41 \\
\hline SM34:2 & $17.8(13 ; 23.5)$ & $14.6(7.8 ; 27.8)$ & $13.2(10.8 ; 20.1)$ & 0.017 & 0.043 & 0.012 & 0.823 \\
\hline SM36:1 & $23.4(16.4 ; 33.5)$ & $22(14.8 ; 32.3)$ & $19.6(11.9 ; 26.4)$ & 0.345 & 0.523 & 0.185 & 0.623 \\
\hline SM36:2 & $9.6(7.4 ; 14.7)$ & $8.8(6.4 ; 18.8)$ & $8.5(4.9 ; 13.1)$ & 0.361 & 0.652 & 0.231 & 0.482 \\
\hline SM38:1 & $23.2(15.8 ; 28.2)$ & $17.9(12.3 ; 26.5)$ & $16.9(12.2 ; 21.7)$ & 0.006 & 0.04 & 0.006 & 0.286 \\
\hline SM39:1 & $9.2(5.9 ; 11.7)$ & $6.8(4.3 ; 11.3)$ & $5.6(4.3 ; 10.6)$ & 0.005 & 0.028 & 0.004 & 0.499 \\
\hline SM39:2 & $1.2(0.5 ; 2.2)$ & $1(0.3 ; 2.2)$ & $0.9(0.3 ; 1.8)$ & 0.371 & 0.435 & 0.29 & 0.566 \\
\hline SM40:1 & $42.6(31.7 ; 59.8)$ & $33.7(25.9 ; 47.4)$ & $32(22.2 ; 44.8)$ & 0.004 & 0.015 & 0.004 & 0.697 \\
\hline SM40:2 & $28(18.6 ; 35.8)$ & $23.1(16.4 ; 36.1)$ & $21(16.9 ; 37.2)$ & 0.017 & 0.026 & 0.022 & 0.681 \\
\hline SM41:1 & $22.2(17 ; 32.9)$ & $19.3(9.7 ; 28.3)$ & $15.4(13.3 ; 21.9)$ & 0.001 & 0.016 & 0.001 & 0.314 \\
\hline SM41:2 & $14(11.6 ; 23.1)$ & $12(7.3 ; 17.3)$ & $11.8(8 ; 20.6)$ & 0.049 & 0.043 & 0.065 & 0.823 \\
\hline SM42:1 & $39.6(25.8 ; 53.1)$ & $29.8(19 ; 42.5)$ & $25.8(19.1 ; 40.2)$ & 0.005 & 0.03 & 0.005 & 0.327 \\
\hline SM42:2 & $66.4(51.6 ; 82.8)$ & $53.6(40.9 ; 77.6)$ & $55.3(38.4 ; 79.6)$ & 0.016 & 0.018 & 0.026 & 0.823 \\
\hline SM42:3 & $22.8(16.7 ; 33.4)$ & $21.4(15.3 ; 30.9)$ & $21.5(13.6 ; 36.8)$ & 0.38 & 0.324 & 0.326 & 0.811 \\
\hline Total Cer/HexCer & $12.2(4.9 ; 20.2)$ & $8.5(2.8 ; 11.7)$ & $5.4(3.1 ; 7.4)$ & $<0.001$ & 0.013 & 0.001 & 0.026 \\
\hline Cer(d18:1/24:0) & $2.2(1.4 ; 4.9)$ & $1.7(1 ; 2.6)$ & $1.6(0.7 ; 2.7)$ & 0.041 & 0.04 & 0.049 & 0.932 \\
\hline HexCer(d18:1/22:0) & $2.8(0.9 ; 5)$ & $1.9(0.3 ; 3)$ & $1.1(0.3 ; 1.7)$ & $<0.001$ & 0.033 & 0.001 & 0.01 \\
\hline HexCer(d18:1/23:0) & $1.5(0.5 ; 2.9)$ & $0.8(0.2 ; 1.7)$ & $0.6(0.2 ; 1.1)$ & 0.001 & 0.016 & 0.001 & 0.117 \\
\hline
\end{tabular}


Table 3 (continued)

\begin{tabular}{|c|c|c|c|c|c|c|c|}
\hline \multirow[t]{2}{*}{ Lipids, $\mu \mathrm{mol} / \mathrm{l}$} & \multirow{2}{*}{$\frac{\text { Healthy subjects }(\mathrm{n}=14)}{\text { Median(Min; Max })}$} & \multirow{2}{*}{$\frac{\operatorname{CAD}(\mathrm{n}=18)}{\operatorname{Median}(\operatorname{Min} ; \operatorname{Max})}$} & \multirow{2}{*}{$\frac{\operatorname{AMI}(\mathrm{n}=17)}{\operatorname{Median}(\operatorname{Min} ; \operatorname{Max})}$} & \multirow[t]{2}{*}{ Kruskal-Wallis, adj. $p$ values } & \multicolumn{3}{|c|}{ Mann-Whitney $\mathrm{U}$, adj. $p$ values } \\
\hline & & & & & $\mathrm{H}$ vs $\mathrm{CAD}$ & $\mathrm{H}$ vs ACS & CAD vs ACS \\
\hline HexCer(d18:1/24:0) & $3.5(1.6 ; 5.7)$ & $2.2(0.3 ; 3.8)$ & $1.3(0.7 ; 2.3)$ & $<0.001$ & 0.012 & $<0.001$ & 0.022 \\
\hline HexCer(d18:1/24:1) & $2.2(0.5 ; 2.9)$ & $1.3(0.5 ; 2.5)$ & $0.9(0.3 ; 1.7)$ & 0.004 & 0.04 & 0.004 & 0.185 \\
\hline \multicolumn{8}{|l|}{ S1P species } \\
\hline $16: 1-S 1 P$ & $0.12(0.09 ; 0.15)$ & $0.1(0.05 ; 0.17)$ & $0.1(0.07 ; 0.13)$ & 0.006 & 0.014 & 0.009 & 0.952 \\
\hline $17: 1-S 1 P$ & $0.03(0.02 ; 0.05)$ & n.d. & n.d. & - & - & - & - \\
\hline 18:1-S1P & $0.55(0.5 ; 0.81)$ & $0.5(0.38 ; 0.82)$ & $0.44(0.29 ; 0.79)$ & 0.011 & 0.096 & 0.013 & 0.11 \\
\hline $18: 0-S 1 P$ & $0.05(0.03 ; 0.07)$ & $0.04(0.03 ; 0.09)$ & $0.03(0.02 ; 0.14)$ & 0.198 & 0.248 & 0.159 & 0.523 \\
\hline \multicolumn{8}{|c|}{ Sphingoid bases of sphingolipids } \\
\hline C16SO & $31.91(14.84 ; 46.01)$ & $24.97(13.24 ; 58.88)$ & $21.05(9.48 ; 48.36)$ & 0.011 & 0.06 & 0.004 & 0.78 \\
\hline C16SA & $0.73(0.3 ; 1.38)$ & $0.63(0.21 ; 1.26)$ & $0.46(0.24 ; 1.13)$ & 0.011 & 0.263 & 0.006 & 0.139 \\
\hline C17SO & $13.49(8.45 ; 22.2)$ & $9.65(3.43 ; 17.94)$ & $9(4.75 ; 23.95)$ & 0.003 & 0.016 & 0.003 & 0.485 \\
\hline C18SO & $145.13(104.41 ; 203.95)$ & $110.45(64.43 ; 177.81)$ & $105.05(67.5 ; 160.82)$ & $<0.001$ & 0.002 & 0.001 & 0.475 \\
\hline C18SA & $4.5(3.11 ; 6.77)$ & $3.2(1.28 ; 10.65)$ & $3.02(1.68 ; 9.48)$ & 0.001 & 0.012 & 0.001 & 0.544 \\
\hline C18SAdiene & $52.44(30.66 ; 78.94)$ & $37.15(24.66 ; 74.43)$ & $35.66(23.3 ; 48.22)$ & 0.005 & 0.043 & 0.003 & 0.501 \\
\hline C19SO & $3.79(1.66 ; 7.28)$ & $4.09(1.33 ; 8.66)$ & $3.12(1.2 ; 6.81)$ & 0.378 & 0.932 & 0.269 & 0.386 \\
\hline C20SO & $0.24(0.14 ; 0.35)$ & $0.23(0.12 ; 0.55)$ & $0.21(0.09 ; 0.36)$ & 0.648 & 0.628 & 0.433 & 0.904 \\
\hline C20SA & $0.02(0.01 ; 0.04)$ & $0.02(0.01 ; 0.03)$ & $0.02(0.01 ; 0.04)$ & 0.747 & 0.911 & 0.805 & 0.509 \\
\hline doxSO & $0.2(0.15 ; 0.7)$ & $0.17(0.08 ; 0.44)$ & $0.19(0.07 ; 0.78)$ & 0.353 & 0.248 & 0.39 & 0.697 \\
\hline doxSA & $0.1(0.07 ; 0.24)$ & $0.09(0.03 ; 0.24)$ & $0.07(0.03 ; 0.33)$ & 0.238 & 0.208 & 0.211 & 0.762 \\
\hline
\end{tabular}
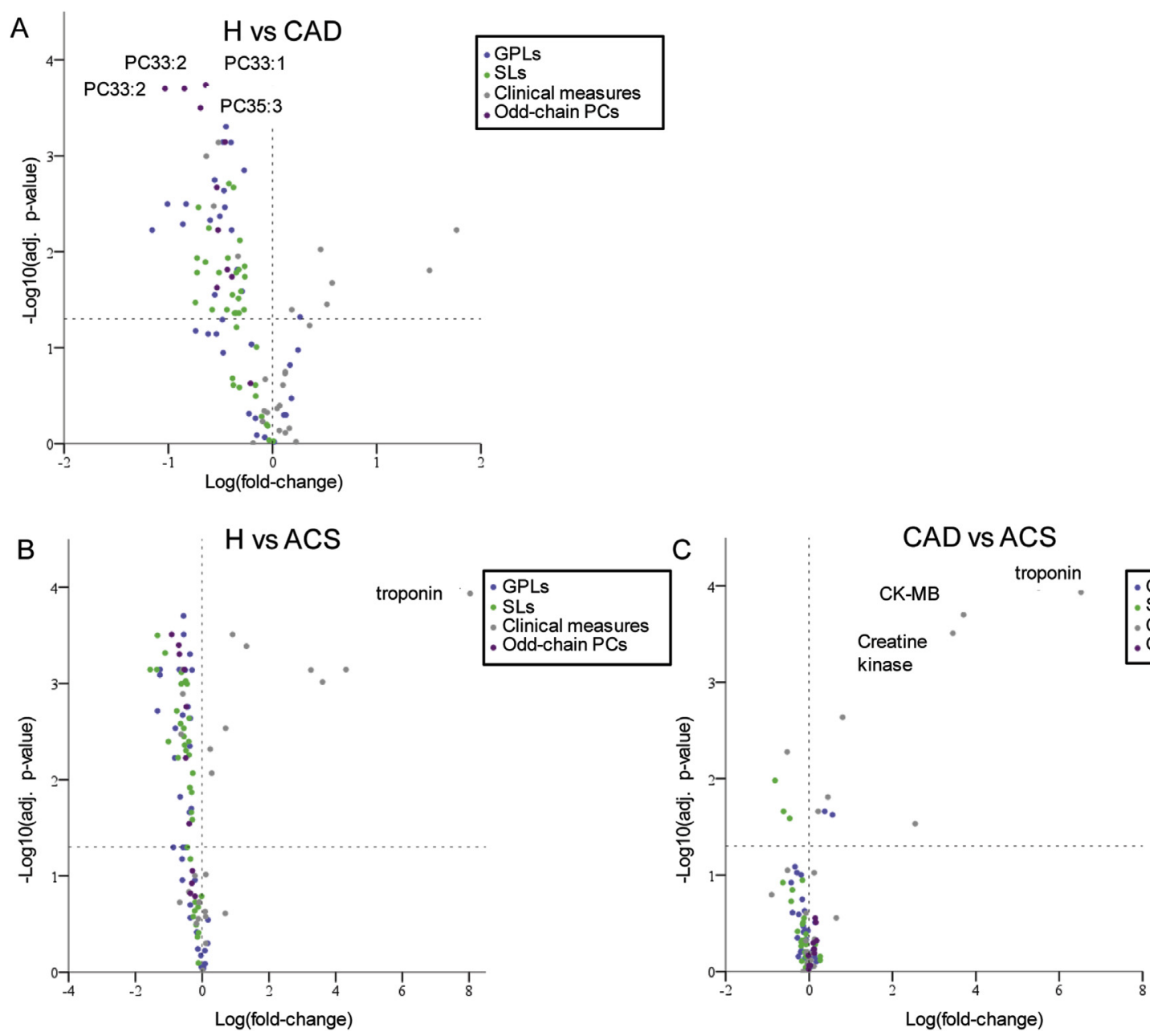

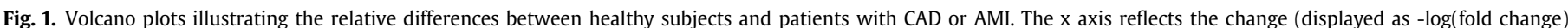

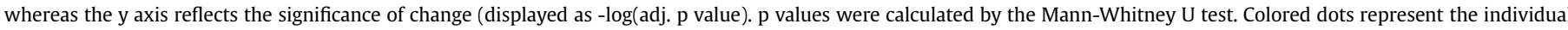

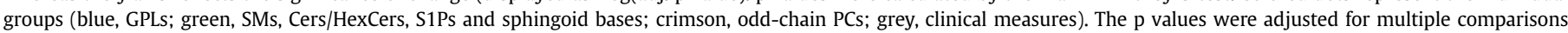

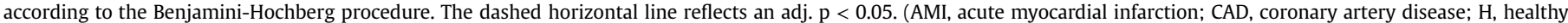

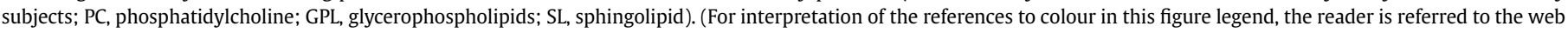
version of this article.) 


\begin{tabular}{c|c|c|c}
\hline Lipids & $\begin{array}{c}\text { Total } \\
\text { cholesterol }\end{array}$ & HDL-C & LDL-C \\
\hline Total PC & 0.81 & 0.66 & 0.68 \\
\hline PC32:0 & 0.58 & 0.65 & 0.45 \\
PC32:1 & 0.49 & 0.51 & 0.42 \\
\hline PC33:1 & 0.72 & 0.8 & 0.57 \\
PC33:2 & 0.63 & 0.71 & 0.5 \\
\hline PC33:3 & 0.57 & 0.75 & 0.43 \\
PC34:0 & 0.64 & 0.59 & 0.52 \\
PC34:1 & 0.57 & 0.64 & 0.44 \\
PC34:2 & 0.68 & 0.5 & 0.6 \\
\hline PC34:3 & 0.64 & 0.61 & 0.54 \\
PC35:1 & 0.72 & 0.57 & 0.66 \\
PC35:2 & 0.66 & 0.61 & 0.56 \\
PC35:3 & 0.66 & 0.68 & 0.52 \\
PC35:4 & 0.44 & 0.45 & 0.36 \\
PC35:5 & 0.48 & 0.54 & 0.39 \\
PC36:0 & 0.55 & 0.42 & 0.46 \\
PC36:1 & 0.73 & 0.53 & 0.68 \\
PC36:2 & 0.55 & 0.33 & 0.49 \\
PC36:3 & 0.72 & 0.43 & 0.62 \\
\hline PC36:4 & 0.13 & 0.1 & 0.12 \\
PC36:5 & 0.27 & 0.48 & 0.17 \\
\hline PC37:4 & 0.48 & 0.38 & 0.43 \\
\hline PC37:5 & 0.44 & 0.56 & 0.3 \\
\hline PC37:6 & 0.53 & 0.61 & 0.43 \\
PC38:3 & 0.42 & -0.18 & 0.44 \\
\hline PC38:4 & 0.08 & -0.16 & 0.1 \\
\hline PC38:5 & 0.33 & 0.35 & 0.24 \\
\hline PC38:6 & 0.1 & 0.01 & 0.07 \\
PC40:5 & 0.29 & 0.11 & 0.25 \\
\hline PC40:6 & 0.06 & -0.18 & 0.07 \\
\hline Total LPC & 0.62 & 0.47 & 0.59 \\
LPC16:0 & 0.81 & 0.53 & 0.74 \\
LPC18:0 & 0.73 & 0.32 & 0.71 \\
\hline LPC18:1 & 0.28 & 0.46 & 0.26 \\
\hline LPC18:2 & 0.01 & 0.12 & 0.04 \\
\hline Total PE & 0.54 & 0.2 & 0.48 \\
PE34:2 & 0.56 & 0.26 & 0.5 \\
PE36:4 & 0.45 & 0.2 & 0.38 \\
PE36:3 & 0.54 & 0.29 & 0.49 \\
PE36:2 & 0.6 & 0.2 & 0.54 \\
PE36:1 & 0.47 & 0.06 & 0.45 \\
PE37:5 & 0.51 & 0.53 & 0.36 \\
PE37:4 & 0.38 & 0.36 & 0.32 \\
PE38:6 & 0.29 & 0.1 & 0.23 \\
PE38:5 & 0.4 & 0.26 & 0.34 \\
PE38:4 & 0.4 & 0.07 & 0.37 \\
\hline & & & \\
\hline & & & \\
\hline
\end{tabular}

\begin{tabular}{cccc}
\hline Lipids & $\begin{array}{c}\text { Total } \\
\text { cholesterol }\end{array}$ & HDL-C & LDL-C \\
\hline Total SM & 0.77 & 0.59 & 0.7 \\
SM32:1 & 0.73 & 0.65 & 0.65 \\
SM34:0 & 0.59 & 0.54 & 0.5 \\
SM34:1 & 0.8 & 0.63 & 0.71 \\
SM34:2 & 0.69 & 0.6 & 0.63 \\
SM36:1 & 0.56 & 0.33 & 0.54 \\
SM36:2 & 0.51 & 0.32 & 0.5 \\
SM38:1 & 0.63 & 0.47 & 0.55 \\
SM39:1 & 0.66 & 0.42 & 0.66 \\
SM40:1 & 0.62 & 0.34 & 0.59 \\
SM40:2 & 0.64 & 0.53 & 0.6 \\
SM41:1 & 0.81 & 0.46 & 0.75 \\
SM41:2 & 0.59 & 0.57 & 0.56 \\
SM42:1 & 0.65 & 0.33 & 0.63 \\
SM42:2 & 0.6 & 0.57 & 0.52 \\
SM42:3 & 0.41 & 0.41 & 0.37 \\
\hline Total Cer/HexCer & 0.78 & 0.35 & 0.75 \\
Cer(d18:1/24:0) & 0.46 & 0.09 & 0.45 \\
HexCer(d18:1/22:0) & 0.76 & 0.27 & 0.75 \\
HexCer(d18:1/23:0) & 0.72 & 0.4 & 0.67 \\
HexCer(d18:1/24:0) & 0.76 & 0.34 & 0.75 \\
HexCer(d18:1/24:1) & 0.65 & 0.36 & 0.58 \\
\hline S1P species & & & \\
16:1-S1P & 0.6 & 0.54 & 0.55 \\
18:1-S1P & 0.44 & 0.2 & 0.41 \\
18:0-S1P & 0.28 & 0.06 & 0.29 \\
\hline Sphingoid bases of sphingolipids & & \\
C16SO & 0.77 & 0.47 & 0.75 \\
C16SA & 0.8 & 0.26 & 0.8 \\
C17SO & 0.85 & 0.51 & 0.81 \\
C18SO & 0.93 & 0.6 & 0.86 \\
C18SA & 0.82 & 0.47 & 0.77 \\
C18SAdiene & 0.78 & 0.55 & 0.73 \\
C19SO & 0.63 & 0.08 & 0.7 \\
C20SO & 0.57 & 0.21 & 0.59 \\
C20SA & 0.47 & -0.06 & 0.55 \\
doxSO & 0.35 & 0.14 & 0.31 \\
doxSA & 0.44 & 0.03 & 0.43 \\
\hline & & & \\
\hline
\end{tabular}

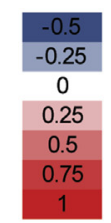


A

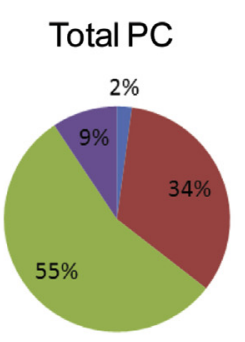

B

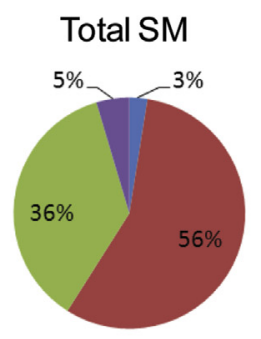

C

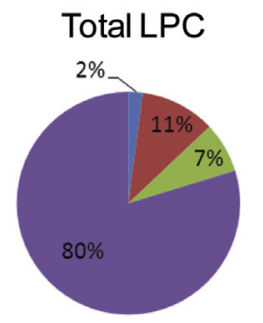

D

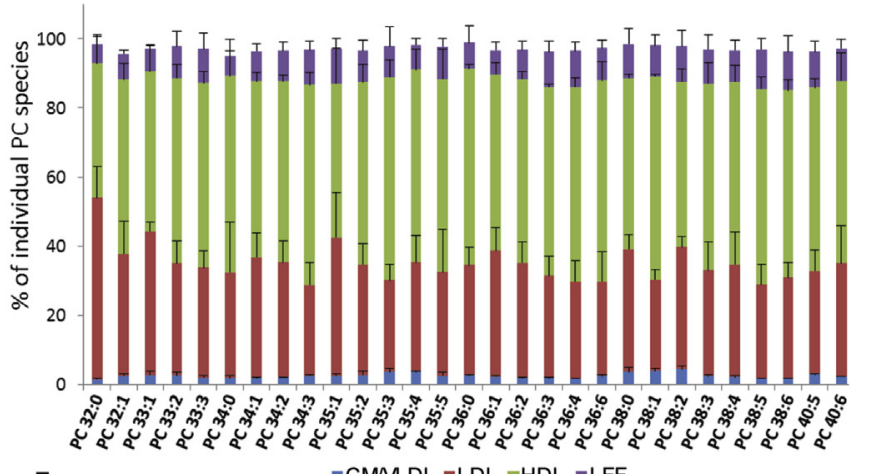

E

$=\mathrm{CMMLL}=\mathrm{LDL}=\mathrm{HDL}$ =LFF
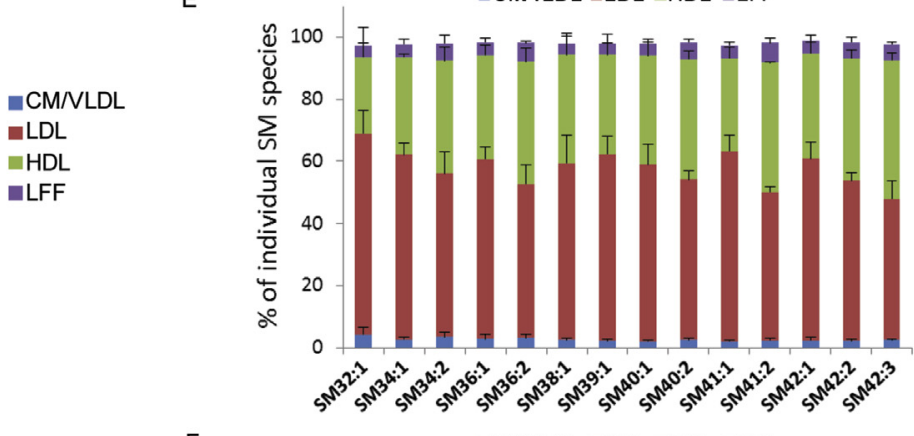

$\mathrm{F}$

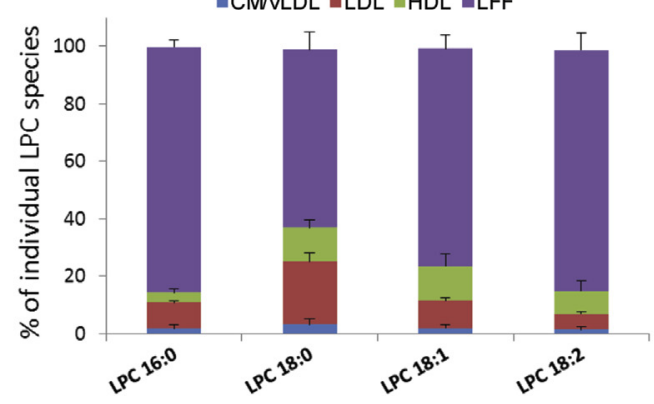

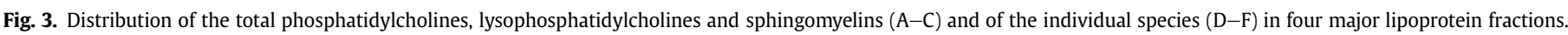

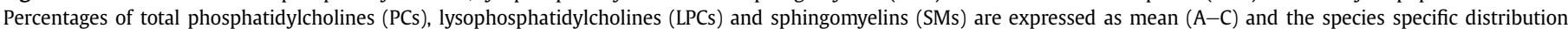

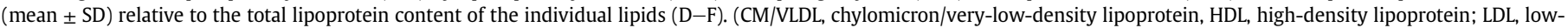
density lipoprotein; LFF, lipoprotein-free fraction).

$[11,27]$. Comparing the individual lipoprotein fractions showed that PC33:1, PC33:2, PC33:3 and PC35:3 are largely associated with HDL which partly explains the strong positive correlations of these lipids with HDL-C as seen by us and others [28].

The physiological functions of plasmalogens are poorly understood. It is unclear whether decreased levels of PC plasmalogens are due to metabolic effects in CAD and AMI or whether plasmalogens play an active role in the pathogenesis of CAD. Plasmalogens possess antioxidant properties due to their ability to scavenge oxygen radicals [29]. Evidence from in vitro studies indicated that plasmalogens are capable of reducing the oxidation of cell membrane cholesterol, polyunsaturated fatty acids and LDL [30-32]. Thus, low levels of PC plasmalogens may be indicators of increased oxidative stress which may explain why PC plasmalogens, but not polyunsaturated PCs, were lower in patients with CAD and AMI.

Our analysis of the sphingoid base profile indicated a reduction of certain sphingoid bases in patients with CAD and AMI. This is in line with significantly reduced plasma levels of certain SM species in patients with CAD and AMI. However, 1-deoxyphingolipids (doxSA and doxSO), recently found as predictive biomarkers for the risk to develop type 2 diabetes mellitus [23,33] were not different between healthy controls and patients with CAD or AMI. Our analysis also revealed the presence of various S1P species, including typical 18:1-S1P 18:1) and atypical 16:1-S1P, 17:1-S1P and 18:0 SA1P. Different S1P species were previously reported in plasma of healthy subjects [34], however, the existence of native 17:1-S1P was here reported for the first time. Among the phosphorylated sphingosines, the canonical S1P (18:1) was the most abundant and found to be lowered in AMI group. In contrast, 16:1S1P, was diminished in plasmas of patients with CAD and AMI compared to healthy subjects. Also other studies reported reduced S1P levels in plasma and HDL of patients with CAD [22,35] as well as a significant inverse association of S1P (18:1) and SA1P (18:0) levels in the HDL-containing fraction of serum with the occurrence of ischemic heart disease [36]. In our study we did not see any significant association between plasma 18:0-SA1P levels and CAD or AMI. Canonical S1P (18:1) is an important regulator of vascular function as well as immune and inflammation responses. HDL is the major carrier of S1P in plasma and HDL bound S1P was reported to mediate several atheroprotective properties HDL [36], [37]. Therefore, differences in the concentration and composition of S1Ps in plasmas of healthy controls and patients with either acute or 
chronic cardiovavascular disease may reflect differences in atheroprotective plasma functions.

\subsection{Limitations}

This study was designed as a purely explorative approach to identify novel lipid signatures in patients with CAD and AMI. Due to the small group sizes it was not possible to adjust for all potential confounders such as HDL-C, LDL-C, TG or medications without the risk of over fitting. This might introduce a bias in particular for the observed associations with HDL-C which is generally lower in the CAD patients. However, not all analyzed lipid species showed the same association with HDL-C indicating that changes in total HDL-C plasma levels are also associated with alterations in the lipid composition of HDL. Further lipid species might show significant changes when validating the study in a bigger cohort.

\section{Conclusions and perspectives}

In conclusion, we found that plasma levels of various glycerophospholipid and sphingolipid species are reduced in patients with CAD and AMI. Among them, PC33:1, PC33:2, PC33:3 and PC35:3, identified as plasmalogens PC(P-18:0/18:2), PC(P-18:0/ $16: 0), \mathrm{PC}(\mathrm{P}-16: 0 / 18: 1)$ and $\mathrm{PC}(\mathrm{P}-16: 0 / 18: 2)$, were the most significantly altered species in the plasma of patients with CAD. Reduced plasmalogen levels might be indicators for oxidative stress as it is typically seen in patients with CAD. We also found that these PC species are preferentially localized in HDL particles and correlate positively with HDL-C levels. However, as this was an explorative study to find novel lipid signatures the observed associations need to be validated in a larger cohort which allows to adjust for more potential confounders in multivariate statistical tests.

\section{Disclosures}

The authors have no conflict of interest pertinent to the subject of this study.

\section{Funding}

TH received a grant from the Zurich Center of Integrated Human Physiology, University of Zurich (ZIHP). AvE was supported by the Foundation for Pathobiochemistry and Molecular Diagnostics of the German Society for Clinical Chemistry and Laboratory Medicine. The SPUM-ACS study (RK, UL, DH, NR, FM, SW, CMM, TFL) was funded by the Swiss National Science Foundation (SPUM 33CM30124112); Swiss Heart Foundation; Fondation Leducq and the Foundation for Cardiovascular Research, Zurich. The SPUM consortium was supported by Roche, Eli Lilly (USA), AstraZeneca, Medtronic, MSD, Sanofi, St. Jude Medical (all Switzerland).

\section{Acknowledgments}

The authors thank all physicians and nurses involved in the SPUM-ACS study and the participants for their contribution to the study and blood sample donation. The authors wish to thank Silvia Radosavljevic for the isolation of the lipoprotein fractions.

\section{References}

[1] M. Nichols, N. Townsend, P. Scarborough, M. Rayner, Cardiovascular disease in Europe 2014: epidemiological update, Eur. Heart J. 35 (42) (Nov 7 2014) 2929.

[2] P. Libby, P.M. Ridker, G.K. Hansson, Progress and challenges in translating the biology of atherosclerosis, Nature 473 (7347) (May 19 2011) 317-325.

[3] F. Crea, G. Liuzzo, Pathogenesis of acute coronary syndromes, J. Am. Coll. Cardiol. 61 (1) (Jan 8 2013) 1-11.
[4] K. Ekroos, M. Janis, K. Tarasov, R. Hurme, R. Laaksonen, Lipidomics: a tool for studies of atherosclerosis, Curr. Atheroscler. Rep. 12 (4) (Jul 2010) 273-281.

[5] T. Hornemann, T.S. Worgall, Sphingolipids and atherosclerosis, Atherosclerosis 226 (1) (Jan 2013) 16-28.

[6] P. Wiesner, K. Leidl, A. Boettcher, G. Schmitz, G. Liebisch, Lipid profiling of FPLC-separated lipoprotein fractions by electrospray ionization tandem mass spectrometry, J. Lipid Res. 50 (3) (Mar 2009) 574-585.

[7] M. Dashti, W. Kulik, F. Hoek, E.C. Veerman, M.P. Peppelenbosch, F. Rezaee, A phospholipidomic analysis of all defined human plasma lipoproteins, Sci. Rep. 1 (2011) 139.

[8] S.M. Hammad, J.S. Pierce, F. Soodavar, et al., Blood sphingolipidomics in healthy humans: impact of sample collection methodology, J. Lipid Res. 51 (10) (Oct 2010) 3074-3087.

[9] P.J. Meikle, G. Wong, D. Tsorotes, et al., Plasma lipidomic analysis of stable and unstable coronary artery disease, Arterioscler. Thromb. Vasc. Biol. 31 (11) (Nov 2011) 2723-2732.

[10] M. Nishimukai, R. Maeba, A. Ikuta, et al., Serum choline plasmalogens-those with oleic acid in sn-2-are biomarkers for coronary artery disease, Clin. Chim. Acta 437 (Nov 1 2014) 147-154.

[11] W. Pruzanski, E. Stefanski, F.C. de Beer, M.C. de Beer, A. Ravandi, A. Kuksis, Comparative analysis of lipid composition of normal and acute-phase high density lipoproteins, J. Lipid Res. 41 (7) (Jul 2000) 1035-1047.

[12] C.E. Kostara, A. Papathanasiou, N. Psychogios, et al., NMR-based lipidomic analysis of blood lipoproteins differentiates the progression of coronary heart disease, J. Proteome Res. 13 (5) (May 2 2014) 2585-2598.

[13] I. Sutter, S. Velagapudi, A. Othman, et al., Plasmalogens of high-density lipoproteins (HDL) are associated with coronary artery disease and anti-apoptotic activity of HDL, Atherosclerosis 241 (2) (Aug 2015) 539-546.

[14] A. Kontush, M.J. Chapman, Lipidomics as a tool for the study of lipoprotein metabolism, Curr. Atheroscler. Rep. 12 (3) (May 2010) 194-201.

[15] C. Stegemann, I. Drozdov, J. Shalhoub, et al., Comparative lipidomics profiling of human atherosclerotic plaques, Circ. Cardiovasc Genet. 4 (3) (Jun 2011) $232-242$.

[16] Y.A. Hannun, L.M. Obeid, Many ceramides, J. Biol. Chem. 286 (32) (Aug 12 2011) 27855-27862.

[17] A. Penno, M.M. Reilly, H. Houlden, et al., Hereditary sensory neuropathy type 1 is caused by the accumulation of two neurotoxic sphingolipids, J. Biol. Chem. 285 (15) (Apr 9 2010) 11178-11187.

[18] N.C. Zitomer, T. Mitchell, K.A. Voss, et al., Ceramide synthase inhibition by fumonisin B1 causes accumulation of 1-deoxysphinganine: a novel category of bioactive 1-deoxysphingoid bases and 1-deoxydihydroceramides biosynthesized by mammalian cell lines and animals, J. Biol. Chem. 284 (8) (Feb 20 2009) 4786-4795.

[19] H. Reiser, R. Klingenberg, D. Hof, et al., Circulating FABP4 is a prognostic biomarker in patients with acute coronary syndrome but not in asymptomatic individuals, Arterioscler. Thromb. Vasc. Biol. 35 (8) (Aug 2015) 1872-1879.

[20] C. Cavelier, L. Rohrer, A. von Eckardstein, ATP-Binding cassette transporter A1 modulates apolipoprotein A-I transcytosis through aortic endothelial cells, Circ. Res. 99 (10) (Nov 10 2006) 1060-1066.

[21] P. Haimi, A. Uphoff, M. Hermansson, P. Somerharju, Software tools for analysis of mass spectrometric lipidome data, Anal. Chem. 78 (24) (Dec 15 2006) 8324-8331.

[22] I. Sutter, R. Park, A. Othman, et al., Apolipoprotein M modulates erythrocyte efflux and tubular reabsorption of sphingosine-1-phosphate, J. Lipid Res. 55 (8) (Jun 20 2014) 1730-1737.

[23] A. Othman, M.F. Rutti, D. Ernst, et al., Plasma deoxysphingolipids: a novel class of biomarkers for the metabolic syndrome? Diabetologia 55 (2) (Feb 2012) 421-431.

[24] D. Yekutieli, Y. Benjamini, Resampling-based false discovery rate controlling multiple test procedures for correlated test statistics, J. Stat. Plan. Inference 82 (1-2) (Dec 1 1999) 171-196.

[25] J. Graessler, D. Schwudke, P.E. Schwarz, R. Herzog, A. Shevchenko, S.R. Bornstein, Top-down lipidomics reveals ether lipid deficiency in blood plasma of hypertensive patients, PLoS One. 4 (7) (2009) e6261.

[26] K.H. Pietilainen, M. Sysi-Aho, A. Rissanen, et al., Acquired obesity is associated with changes in the serum lipidomic profile independent of genetic effects-a monozygotic twin study, PLoS One. 2 (2) (2007) e218.

[27] P.P. Laurila, I. Surakka, A.P. Sarin, et al., Genomic, transcriptomic, and lipidomic profiling highlights the role of inflammation in individuals with low high-density lipoprotein cholesterol, Arterioscler. Thromb. Vasc. Biol. 33 (4) (Apr 2013) 847-857.

[28] R. Maeba, T. Maeda, M. Kinoshita, et al., Plasmalogens in human serum positively correlate with high- density lipoprotein and decrease with aging, J. Atheroscler. Thromb. 14 (1) (Feb 2007) 12-18.

[29] S. Wallner, G. Schmitz, Plasmalogens the neglected regulatory and scavenging lipid species, Chem. Phys. Lipids. 164 (6) (Sep 2011) 573-589.

[30] D. Reiss, K. Beyer, B. Engelmann, Delayed oxidative degradation of polyunsaturated diacyl phospholipids in the presence of plasmalogen phospholipids in vitro, Biochem. J. 323 (Pt 3) (May 1 1997) 807-814.

[31] G. Jurgens, A. Fell, G. Ledinski, Q. Chen, F. Paltauf, Delay of copper-catalyzed oxidation of low density lipoprotein by in vitro enrichment with choline or ethanolamine plasmalogens, Chem. Phys. Lipids 77 (1) (Aug 1 1995) 25-31.

[32] R. Maeba, N. Ueta, Ethanolamine plasmalogens prevent the oxidation of cholesterol by reducing the oxidizability of cholesterol in phospholipid bilayers, J. Lipid Res. 44 (1) (Jan 2003) 164-171. 
[33] M. Bertea, M.F. Rutti, A. Othman, et al., Deoxysphingoid bases as plasma markers in diabetes mellitus, Lipids Health Dis. 9 (2010) 84.

[34] O. Quehenberger, A.M. Armando, A.H. Brown, et al., Lipidomics reveals a remarkable diversity of lipids in human plasma, J. Lipid Res. 51 (11) (Nov 2010) 3299-3305.

[35] K.J. Sattler, S. Elbasan, P. Keul, et al., Sphingosine 1-phosphate levels in plasma and HDL are altered in coronary artery disease, Basic Res. Cardiol. 105 (6) (Nov
2010) 821-832.

[36] K.M. Argraves, A.A. Sethi, P.J. Gazzolo, et al., S1P, dihydro-S1P and C24:1ceramide levels in the HDL-containing fraction of serum inversely correlate with occurrence of ischemic heart disease, Lipids Health Dis. 10 (2011) 70.

[37] T.F. Luscher, U. Landmesser, A. von Eckardstein, A.M. Fogelman, High-density lipoprotein: vascular protective effects, dysfunction, and potential as therapeutic target, Circ. Res. 114 (1) (Jan 3 2014) 171-182. 